\title{
Promoter methylation of RNF180 is associated with H.pylori infection and serves as a marker for gastric cancer and atrophic gastritis
}

\author{
Fang Han ${ }^{1, *}$, Li-ping Sun ${ }^{1, *}$, Shuang Liuㄹ, Qian $\mathrm{Xu}^{1}$, Qiao-yi Liang ${ }^{2}$, Zhe Zhang ${ }^{3}$, \\ Hai-chao $\mathrm{Cao}^{3}$, Jun Yu², Dai-ming Fan ${ }^{3}$, Yong-zhan $\mathrm{Nie}^{3}$, Kai-chun Wu ${ }^{3}$, Yuan Yuan ${ }^{1}$ \\ ${ }^{1}$ Tumor Etiology and Screening Department of Cancer Institute and General Surgery, The First Affiliated Hospital of China \\ Medical University, and Key Laboratory of Cancer Etiology and Prevention (China Medical University), Liaoning Provincial \\ Education Department, Shenyang, Liaoning, China \\ ${ }^{2}$ Institute of Digestive Disease and Department of Medicine and Therapeutics, State Key Laboratory of Digestive Disease, \\ Li Ka Shing Institute of Health Sciences, CUHK Shenzhen Research Institute, The Chinese University of Hong Kong, Hong Kong \\ ${ }^{3}$ State Key Laboratory of Cancer Biology and Xijing Hospital of Digestive Diseases, Xijing Hospital, Fourth Military Medical \\ University, Xi'an, Shanxi, China \\ *These authors have contributed equally to this work
}

Correspondence to: Yuan Yuan, e-mail: yyuan@mail.cmu.edu.cn Kai-chun Wu, e-mail: kaicwu@fmmu.edu.cn

Keywords: gastric cancer, atrophic gastritis, bisulfite genomic sequencing, CpG islands, methylation

Received: October 17, 2015

Accepted: March 06, 2016

Published: April 01, 2016

\section{ABSTRACT}

Promoter methylation (PM) of RING-finger protein (RNF) 180 affects gastric cancer (GC) prognosis, but its association with risk of GC or atrophic gastritis (AG) is unclear. We investigated relationships between RNF180 PM and GC or AG, and the effects of Helicobactor pylori (H. pylori) infection on RNF180 PM. This study included 513 subjects (159 with GC, 186 with AG, and 168 healthy controls [CON]) for RNF180 PM analysis, and another 55 GC patients for RNF180 gene expression analysis. Methylation was quantified using average methylation rates (AMR), methylated CpG site counts (MSC) and hypermethylated CpG site counts (HSC). RNF180 promoter AMR and MSC increased with disease severity. Optimal cut-offs were GC + AG: AMR $>0.153$, MSC $>4$ or HSC $>1$; GC: AMR $>0.316$, MSC $>15$ and HSC $>6$. Hypermethylation at 5 CpG sites differed significantly between GC/AG and CON groups, and was more common in GC patients than AG and CON groups for 2 other CpG sites. The expression of RNF180 mRNA levels in tumor were significantly lower than those in non-tumor, with the same as in hypermethylation than hypomethylation group. H.pylori infection increased methylation in normal tissue or mild gastritis, and increased hypermethylation risk at 3 CpG sites in AG. In conclusion, higher AMR, MSC and HSC levels could identify AG + GC or GC. Some RNF180 promoter CpG sites could identify precancerous or early-stage GC. H.pylori affects RNF180 PM in normal tissue or mild gastritis, and increases hypermethylation in 3 CpG sites in AG.

\section{INTRODUCTION}

Aberrant $\mathrm{CpG}$ island methylation, especially in promoter regions of tumor suppressor genes, is related to tumorigenesis. For example, RUNX3 promoter methylation (PM) is related to esophageal squamous cell carcinoma [1]. WIF1 and DKK3 to poor prognosis in breast cancer [2]; FGR3 mutation and hypermethylation to bladder cancer [3] and SFRP2 hypermethylation to gastric cancer (GC) $[4,5]$. CpG island methylation can inactivate or downregulate tumor suppressor genes, ultimately leading to tumor development and progression. In recent years, relationships between the PM of tumor suppressor genes and carcinoma risk have attracted much attention in methylation research $[6,7]$.

Really Interesting, new gene (RING) Finger (RNF) is a family of ubiquitin ligases that function as tumor suppressors [8-10]. RNF180 is a recently discovered 
member of the RNF family - an E3 ubiquitin ligase that reportedly participates in proliferation and differentiation [11]. Although the PM status of RNF180 is known to affect GC prognosis [12-14]; however, its effect on risk of $\mathrm{GC}$, or on its most important precancerous state, atrophic gastritis (AG), remain unclear.

Helicobactor pylori (H.pylori) is a major carcinogen of gastric cancer and can also lead to abnormal DNA methylation [15-19]. Whether H.pylori infection would modify RNF180 DNA methylation has not been revealed yet. This study focused on the relationships between RNF180 PM and risk of GC and AG, and the effect of H.pylori infection on RNF180 PM.

\section{RESULTS}

\section{Relationship between AMR of RNF180 promoter and GC or AG}

We amplified the $R N F 180$ promoter area from -224 bp to +94 bp (a 318-bp fragment). The results show AMR in the $R N F 180$ promoter to increase with disease severity, such that $\mathrm{CON}<\mathrm{AG}<\mathrm{GC}(\mathrm{GC}$ vs. AG: $P<0.021 ; \mathrm{AG}$ vs. CON: $P<0.001$; Figure 1A).

We also evaluated the validity of $R N F 180$ promoter area AMR in distinguishing AG or GC from controls, by $\mathrm{ROC}$ curves. We found that $\mathrm{AMR}>0.153$ was $78.6 \%$ sensitive and $77.4 \%$ specific $(A U C=0.802)$ in distinguishing $[\mathrm{GC}+\mathrm{AG}]$ from controls (Figure 2A); and at 0.316 , was $41.5 \%$ sensitive and $87.9 \%$ specific for GC vs. $[\mathrm{AG}+\mathrm{CON}]$ (Figure $2 \mathrm{~B})$.

\section{Relationships between MSC and HSC of the RNF180 promoter area and GC or AG}

Relationships between MSC or HSC and GC or AG were analyzed by the Mann-Whitney $U$ test. As with AMR, MSC increased with disease severity (i.e., CON < $\mathrm{AG}<\mathrm{GC}$, Figure 1B). However, HSC in the GC and AG groups were higher than for controls, $(P<0.001$ for both $)$, but did not significantly differ between the GC and AG groups $(P=0.301$; Figure $1 \mathrm{C})$.

We further evaluated $R N F 180$ promoter MSC and HSC as biomarkers for AG or GC, using ROC curves. We found MSC > 4 was $78.6 \%$ sensitive and $77.4 \%$ specific $(A U C=0.772)$ in distinguishing $[\mathrm{GC}+\mathrm{AG}]$ groups from controls (Figure 2C); $\mathrm{MSC}>15$ was $48.4 \%$ sensitive and $87.9 \%$ specific $(\mathrm{AUC}=0.772$ ) in distinguishing $\mathrm{GC}$ from $[\mathrm{AG}+\mathrm{CON}]$ (Figure 2D). We found that $\mathrm{HSC}>1$ was $74.5 \%$ sensitive and $83.3 \%$ specific $(\mathrm{AUC}=0.813$ ) in distinguishing $\mathrm{GC}+\mathrm{AG}$ from controls (Figure 2E); and at $\mathrm{HSC}>6$, was $35.2 \%$ sensitive and $92.1 \% \operatorname{specific}(\mathrm{AUC}=0.654)$ at distinguishing GC from [AG + CON] (Figure 2F).

\section{Relationship between hypermethylated of RNF180 promoter CpG sites and GC or AG}

The location of every hypermethylation site in RNF180 gene and distribution of hypermethylation frequency in GC, AG and control groups were shown in Supplementary Table S1, Based on a comparative analysis of relationships between MSC or $\mathrm{HSC}$ and $\mathrm{GC}$ or $\mathrm{AG}$, we selected $8 \mathrm{CpG}$ sites with which hypermethylation frequencies $>25 \%$ among a total 513 individualsM3(-165), M5(-148), M7(-133), M8(-130), M20(-57), M25(-34), M27(-26) and M30(+5) — to evaluate the risk of AG or GC. All results were analyzed using logistic regression. $P$ values and odd ratio (OR) were adjusted by sex, age, smoking, drinking and H.pylori infection.

We found hypermethylation frequencies of M5(-148) and M27(-26) in the GC group were higher than in the $\mathrm{AG}+\mathrm{CON}$ group, and were associated with increased GC risks of 5.85 folds (95\% confidence interval [CI]: 3.20-10.69) and 4.27 folds (95\% CI: 2.54-7.19), respectively. We also found that hypermethylation in $5 \mathrm{CpG}$ sites-M3(-165), M7(-133), M20(-57), M25(-34) and $\mathrm{M} 30(+5)$ - could increase the risks for $\mathrm{AG}$ and $\mathrm{GC}$ and there is significant difference when compares to random two groups of GC AG CON(GC vs. CON, AG vs. CON and GC vs. AG, Table 1).
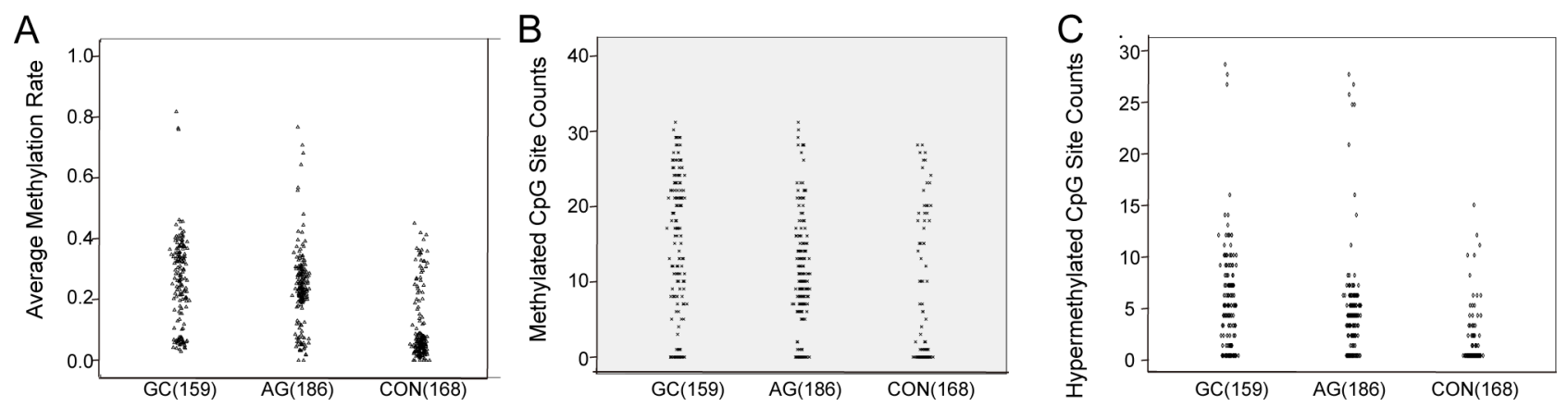

Figure 1: The scatter plot of RNF180 gene promoter methylation. (A) The average methylation rate of $R N F 180$ gene in different gastric diseases; (B) The methylated CpG site counts of $R N F 180$ gene in different gastric diseases; (C) The hypemethylated CpG site counts $R N F 180$ gene in different gastric diseases. 


\section{Relationship of hypermethylation of RNF180 AND RNF180 mRNA expression}

To explore the possible effect of RNF180 promoter methylation on gene expression, we further evaluated the relationship of the hypermethylation and the expression of RNF180 mRNA using the Mann-Whitney $U$ test. In tumor specimens, the RNF180 mRNA levels were significantly lower than those in non-tumor specimens $(P=0.003)$. In addition, the RNF180 mRNA levels were decreased in tend from hypermethylation to hypomethylation, although the $P$ value did not reach the statistical significance $(P=0.075$, Table 2$)$.

\section{Effect of H.pylori infection on RNF180 PM}

To explore the effect on methylation by H.pylori infection, we subdivided the CON, AG and GC groups by their H.pylori infection status, using the Mann-Whitney $U$ test. AMR did not significantly differ between the H.pylori $i^{-}$and H.pylori ${ }^{+}$subgroups for in $\mathrm{GC}(P=0.761)$ or AG $(P=0.581)$, but was higher in H.pylori ${ }^{+}$subgroup than H.pylori subgroup among the controls $(P=0.012)$. A similar pattern was seen in the control group for HSC $\left(P=0.037\right.$; Table 3). In the H.pylori ${ }^{+}$ sub-groups, we found $8 \mathrm{CpG}$ sites with hypermethylation frequency $>25 \%$ of all 513 individuals. The results also
A $G C+A G$ vs. $C O N ., A \cup C=0.802$

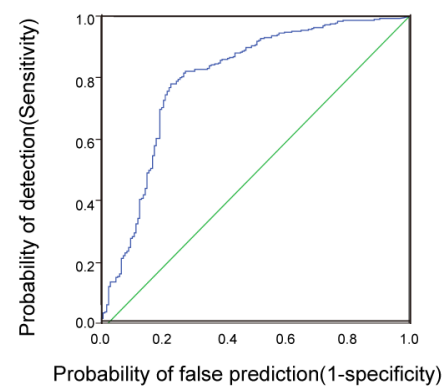

C

Methylated CpG site counts, $\mathrm{GC}+\mathrm{AG}$ vs. Con. , $\mathrm{AUC}=0.772$

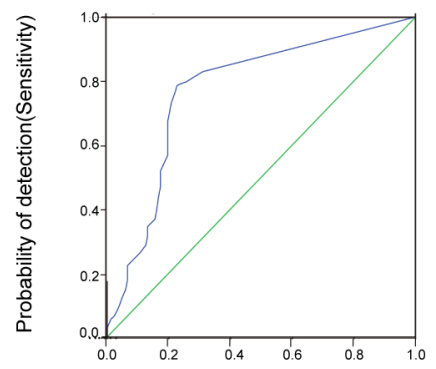

Probability of false prediction(1-specificity)

\section{$E$}

Hypermethylated $\mathrm{CpG}$ site counts, $G C+A G$ vs. $C O N$.,$A \cup C=0.813$

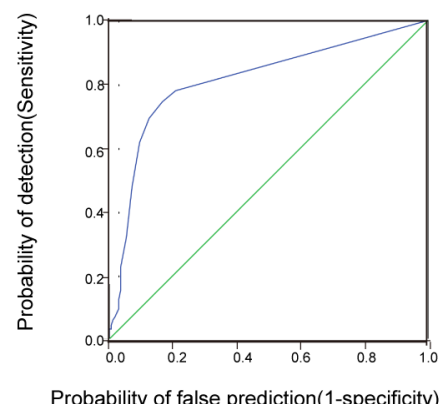

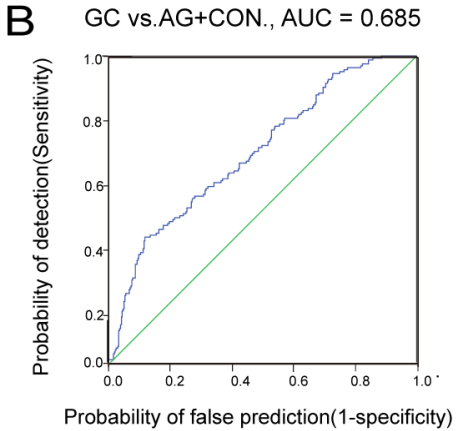

D Methylated CpG site counts, GCvs.AG+ Con. , AUC $=0.677$

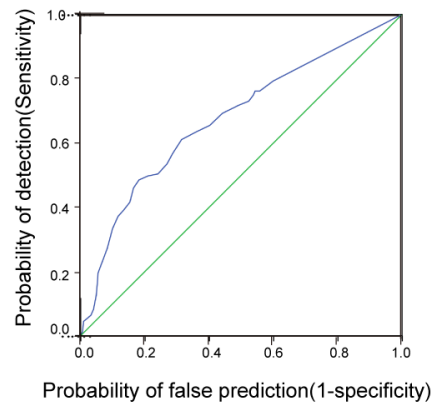

F Hypermethylated CpG site counts, $G C$ vs. $A G+C O N$. $A \cup C=0.654$

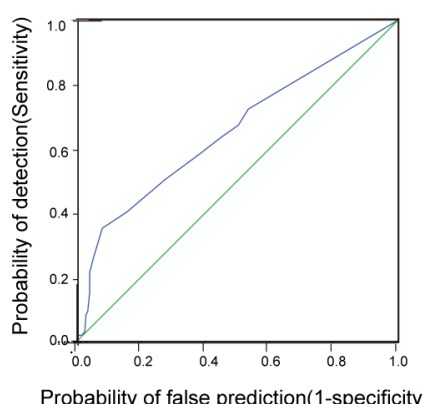

Figure 2: The ROC curve of RNF180 gene promoter methylation. (A) GC + AG vs. CON., AUC = 0.802; (B) GC vs.AG + Con., $\mathrm{AUC}=0.685 ;(\mathbf{C})$ Methylated $\mathrm{CpG}$ site counts, GC + AG vs. CON., AUC = 0.772; (D) Methylated CpG site counts, GC vs. AG + CON., $\mathrm{AUC}=0.677$; (E) Hypermethylated $\mathrm{CpG}$ site counts, $\mathrm{GC}+\mathrm{AG}$ vs. CON., AUC $=0.813$; (F) Hypermethylated CpG site counts, GC vs. AG $+\mathrm{CON}$ AUC $=0.654$. 
Table 1: Association between hypermethylation frequency and risks of gastric cancer and atrophic gastritis

\begin{tabular}{|c|c|c|c|c|c|c|c|c|c|c|c|c|}
\hline & \multirow[b]{2}{*}{ GC } & \multirow[b]{2}{*}{ AG } & \multirow[b]{2}{*}{ CON } & \multicolumn{2}{|c|}{ GC vs CON } & \multicolumn{2}{|c|}{ GC vs AG } & \multicolumn{2}{|c|}{ AG vs CON } & \multicolumn{2}{|c|}{$\mathrm{GC}$ vs $\mathrm{AG}+\mathrm{CON}$} \\
\hline & & & & & $\boldsymbol{P}^{a}$ & $\begin{array}{c}\text { OR } \\
(95 \% \mathrm{CI})\end{array}$ & $P$ & $\begin{array}{c}\text { OR } \\
(95 \% \text { CI })\end{array}$ & $P$ & $\begin{array}{c}\text { OR } \\
(95 \% \mathrm{CI})\end{array}$ & $P$ & $\begin{array}{c}\text { OR } \\
(95 \% \mathrm{CI})\end{array}$ \\
\hline \multirow[t]{2}{*}{ M3 } & $\mathrm{L}^{\mathrm{b}}$ & $101(63.5)$ & $76(40.9)$ & $160(95.2)$ & & 1 (Ref.) & & 1 (Ref.) & & 1 (Ref.) & & 1 (Ref.) \\
\hline & $\mathrm{H}^{\mathrm{c}}$ & $58(36.3)$ & $110(59.1)$ & $8(4.8)$ & $<0.001$ & $\begin{array}{c}11.43 \\
(4.95,26.41)\end{array}$ & $<0.001$ & $0.36(0.22,0.59)$ & $<0.001$ & $\begin{array}{c}25.05 \\
(10.51,59.71)\end{array}$ & 0.981 & $\begin{array}{c}0.98 \\
(0.64,1.51)\end{array}$ \\
\hline \multirow[t]{2}{*}{ M5 } & $\mathrm{L}$ & $120(75.5)$ & $171(92.4)$ & $163(97.0)$ & & 1 (Ref.) & & 1 (Ref.) & & 1 (Ref.) & & 1 (Ref.) \\
\hline & $\mathrm{H}$ & $39(24.5)$ & $14(7.6)$ & $5(3.0)$ & $<0.001$ & $\begin{array}{c}10.28 \\
(3.70,28.56) \\
\end{array}$ & $<0.001$ & $3.86(1.94,7.68)$ & 0.083 & $\begin{array}{c}2.91 \\
(0.87,9.70) \\
\end{array}$ & $<0.001$ & $\begin{array}{c}5.85 \\
(3.20,10.69) \\
\end{array}$ \\
\hline \multirow[t]{2}{*}{ M7 } & $\mathrm{L}$ & $117(73.6)$ & 77 (41.6) & $160(95.2)$ & & 1 (Ref.) & & 1 (Ref.) & & 1 (Ref.) & & 1 (Ref.) \\
\hline & $\mathrm{H}$ & $42(26.4)$ & $108(58.4)$ & $8(4.8)$ & $<0.001$ & $\begin{array}{c}7.26 \\
(3.12,16.89)\end{array}$ & $<0.001$ & $0.23(0.14,0.37)$ & $<0.001$ & $\begin{array}{c}22.70 \\
(9.93,51.95)\end{array}$ & 0.028 & $\begin{array}{c}0.60 \\
(0.38,0.95)\end{array}$ \\
\hline \multirow[t]{2}{*}{ M8 } & $\mathrm{L}$ & $122(76.7)$ & $127(68.6)$ & $160(95.2)$ & & 1 (Ref.) & & 1 (Ref.) & & 1 (Ref.) & & 1 (Ref.) \\
\hline & $\mathrm{H}$ & $37(23.3)$ & $58(31.4)$ & $8(4.8)$ & $<0.001$ & $\begin{array}{c}6.43 \\
(2.74,15.27) \\
\end{array}$ & 0.065 & $0.61(0.37,1.03)$ & $<0.001$ & $\begin{array}{c}9.28 \\
(3.95,21.84) \\
\end{array}$ & 0.406 & $\begin{array}{c}1.23 \\
(0.76,1.98)\end{array}$ \\
\hline \multirow[t]{2}{*}{ M20 } & $\mathrm{L}$ & $111(69.8)$ & $108(58.4)$ & $158(94.0)$ & & 1 (Ref., & & 1 (Ref.) & & 1 (Ref.) & & 1 (Ref.) \\
\hline & $\mathrm{H}$ & $48(30.2)$ & 77 (41.6) & $10(6.0)$ & $<0.001$ & $\begin{array}{c}5.22 \\
(2.42,11.27) \\
\end{array}$ & 0.012 & $0.54(0.33,0.87)$ & $<0.001$ & $\begin{array}{c}7.78 \\
(3.60,16.82) \\
\end{array}$ & 0.608 & $\begin{array}{c}1.13 \\
(0.72,1.76)\end{array}$ \\
\hline \multirow[t]{2}{*}{ M25 } & $\mathrm{L}$ & $78(49.1)$ & $53(28.6)$ & $145(86.3)$ & & 1 (Ref.) & & 1 (Ref.) & & 1 (Ref.) & & 1 (Ref.) \\
\hline & $\mathrm{H}$ & $81(50.9)$ & $132(71.4)$ & $23(13.7)$ & $<0.001$ & $\begin{array}{c}5.82 \\
(3.24,10.47) \\
\end{array}$ & $<0.001$ & $\begin{array}{c}0.411(0.26 \\
0.66)\end{array}$ & $<0.001$ & $\begin{array}{c}10.77 \\
(5.88,19.71) \\
\end{array}$ & 0.326 & $\begin{array}{c}1.23 \\
(0.82,1.84)\end{array}$ \\
\hline \multirow[t]{2}{*}{ M27 } & $\mathrm{L}$ & $113(71.1)$ & $165(89.2)$ & $157(93.5)$ & & 1 (Ref.) & & 1 (Ref.) & & 1 (Ref.) & & 1 (Ref.) \\
\hline & $\mathrm{H}$ & $46(28.9)$ & $20(10.8)$ & $11(6.5)$ & $<0.001$ & $\begin{array}{c}5.29 \\
(2.44,11.44) \\
\end{array}$ & $<0.001$ & $3.16(1.72,5.80)$ & 0.067 & $\begin{array}{c}2.31 \\
(0.94,5.64)\end{array}$ & $<0.001$ & $\begin{array}{c}4.27 \\
(2.54,7.19)\end{array}$ \\
\hline \multirow[t]{2}{*}{ M30 } & $\mathrm{L}$ & $82(51.6)$ & $48(25.9)$ & $150(89.3)$ & & 1 (Ref.) & & 1 (Ref.) & & 1 (Ref.) & & 1 (Ref.) \\
\hline & $\mathrm{H}$ & 77 (48.4) & $137(74.1)$ & $18(10.7)$ & $<0.001$ & $\begin{array}{c}7.13 \\
(3.81,13.36)\end{array}$ & $<0.001$ & $0.31(0.19,0.51)$ & $<0.001$ & $\begin{array}{c}15.28 \\
(8.07,28.93)\end{array}$ & 0.68 & $\begin{array}{c}1.09 \\
(0.73,1.64)\end{array}$ \\
\hline
\end{tabular}

Note: a: $P$ value and odd ratio(OR) were adjusted by sex, age, smoking, drinking and H.pylori infection. $P$ value OR and corresponding $95 \%$ interval confidence were calculated to measure the association between hypermethylation of $\mathrm{CpG}$ site and the risks of different groups. b: the number of hypomethylated cases; c: H:hypermethylation. GC: gastric cancer; AG: atrophic gastritis; CON: control; OR: odd ratio; CI: interval confidence.

Table 2: Relationship between RNF180 expression and gastric diseases, RNF180 hypermethylation

\begin{tabular}{|l|c|c|c|c|}
\hline \multicolumn{2}{c}{ group } & \multicolumn{1}{c}{$\Delta \mathbf{C t}($ Mean \pm SD) } & Normalized 2- $\Delta \mathbf{\Delta C t}$ & $\boldsymbol{P}$ \\
\hline \multirow{2}{*}{ gastric diseases } & non-tumor & $4.58 \pm 1.65$ & $1.0(0.32,3.14)$ & Ref. \\
\cline { 2 - 5 } & tumor & $5.58 \pm 2.00$ & $0.5(0.13,2.00)$ & $\mathbf{0 . 0 0 3}$ \\
\hline \multirow{2}{*}{ methylation } & hypomethylation & $4.99 \pm 1.90$ & $1.0(0.27,3.73)$ & Ref. \\
\cline { 2 - 5 } & hypermethylation & $6.46 \pm 1.50$ & $0.36(0.13,1.02)$ & 0.075 \\
\hline
\end{tabular}

showed that $3 \mathrm{CpG}$ sites-M3(-165) (OR: 2.74; 95\% CI: 1.37-5.47), M25(-34) (OR: 2.62; 95\% CI: 1.29-5.31) and $\mathrm{M} 30(+5)(\mathrm{OR}: 2.80 ; 95 \% \mathrm{CI}: 1.38-5.71)$, had higher hypermethylation risk in the H.pylori ${ }^{+}$AG subgroup; whereas M27(-26) had a higher methylation risk in the H.pylori ${ }^{-}$AG subgroup. The $\mathrm{M} 30(+5)$ site also had a higher methylation risk in the H.pylori ${ }^{+} \mathrm{CON}$ subgroup (Table 4).

\section{DISCUSSION}

Products of tumor suppressor genes regulate various cellular functions, and expression of these products can be modified by DNA methylation [22]. Under methylated conditions, RNA polymerase cannot effectively combine with the promoter and thus transcribe the gene, which can lead to tumorigenesis and development of cancer [23]. RNF180 is a recently discovered suppressor gene; its product, Rines, is a membrane-bound E3 ubiquitin ligase with a coiled-coil domain and RING finger [11]. Function studies discovered the RNF180 product could activate apoptosis by up-regulating the factors TIMP3 and CDK2A. It also could inhibit cell proliferation by upregulating anti-proliferation factors MTSS1 and CDKN2A [12]. Further studies showed RNF180 PM occurred more frequently in $\mathrm{GC}$ tissues, and $R N F 180$ was thus less expressed in patients with GC [13]. RNF180 can inhibit 
Table 3: Relationship between H.pylori infection and DNA methylation of RNF180 promoter area in different gastric diseases

\begin{tabular}{|c|c|c|c|c|c|c|c|c|}
\hline \multirow{2}{*}{ Group } & \multirow{2}{*}{ H.pylori } & \multirow[t]{2}{*}{$n$} & \multicolumn{2}{|l|}{$\begin{array}{c}\text { Average } \\
\text { Methylation Rate }\end{array}$} & \multicolumn{2}{|l|}{$\begin{array}{c}\text { Methylated CpG } \\
\text { sites count }\end{array}$} & \multicolumn{2}{|l|}{$\begin{array}{l}\text { Hypermethylated } \\
\text { CpG sites count }\end{array}$} \\
\hline & & & Median (25th,75th) & $P$ & Median (25th, 75th) & $P$ & Median (25th, 75th) & $P$ \\
\hline \multirow[t]{2}{*}{$\mathrm{GC}$} & $(-)$ & 84 & $0.28(0.15,0.36)$ & \multirow{2}{*}{0.761} & $14(3.5,23.75)$ & \multirow{2}{*}{0.744} & $5(0,8)$ & \multirow{2}{*}{0.697} \\
\hline & $(+)$ & 75 & $0.26(0.17,0.34)$ & & $14(4,21)$ & & $4(0,7)$ & \\
\hline \multirow[t]{2}{*}{$\mathrm{AG}$} & $(-)$ & 55 & $0.23(0.09,0.31)$ & \multirow{2}{*}{0.581} & $9(1,16)$ & \multirow{2}{*}{0.494} & $4(0,6)$ & \multirow{2}{*}{0.634} \\
\hline & $(+)$ & 131 & $0.24(0.21,0.29)$ & & $10(7,14)$ & & $4(3,5)$ & \\
\hline \multirow[t]{2}{*}{$\mathrm{CON}$} & $(-)$ & 143 & $0.05(0.03,0.12)$ & \multirow{2}{*}{0.012} & $0(0,1)$ & \multirow{2}{*}{0.108} & $0(0,0)$ & \multirow{2}{*}{0.037} \\
\hline & $(+)$ & 25 & $0.08(0.51,0.32)$ & & $0(0,19)$ & & $0(0,3)$ & \\
\hline
\end{tabular}

$P$ value for Mann-Whitney $U$ test comparing the difference of variables between H.pylori positive and negative groups. GC: gastric cancer; AG: atrophic gastritis; CON:control.

tumorigenesis, and its methylation is related to poor prognosis $[13,14]$. However, until now, the relationship between the RNF180 PM and the risk of GC or its precancerous condition had not been deeply analyzed. To our knowledge, this is the first report about RNF180 $\mathrm{PM}$ and risk of $\mathrm{GC}$ or $\mathrm{AG}$. We calculated differences in methylation distribution between $\mathrm{CON}, \mathrm{AG}$ and GC groups, to identify $\mathrm{CpG}$ sites with high sensitivity in distinguishing $\mathrm{AG}$ or $\mathrm{GC}$ from normal controls. We explored the relationship of the hypermethylation and the expression of RNF180 mRNA and also analyzed the effect of H.pylori infection on RNF180 PM.

First, we compared differences in AMR among the control group (CON), atrophy gastritis group (AG) and gastric cancer group (GC) and found AMR significantly differed between $\mathrm{CON}, \mathrm{AG}$ and $\mathrm{GC}$, increasing with gastric disease severity $(\mathrm{GC}>\mathrm{AG}>\mathrm{CON})$. We further evaluated the diagnosis efficacy of AMR. At $>0.153$, AMR distinguished GC + AG from controls at 78.6\% sensitivity and $77.4 \%$ specificity ( $\mathrm{AUC}=0.802$ ); at $>0.316$, AMR distinguished GC from $\mathrm{AG}+\mathrm{CON}$ was $41.5 \%$ sensitive and $87.9 \%$ specific $(\mathrm{AUC}=0.685)$.

Reportedly, PM of tumor suppressor genes (RUNX3, $C D H 1, C D H 13, D A P K, G S T P 1$, etc.) may gradually accumulate with the severity of disease and stimulation of environment factors $[24,25]$. We speculate that AG may be part of gastric carcinogenesis, in which RNF180 PM gradually increases, especially the average methylation rate of the $R N F 180$ promoter area. As overall methylation increases over time, RNA polymerase is less able to combine with the promoter area, with expression and translation of $R N F 180$ products down-regulating further, eventually silencing $R N F 180$ [12]. When this process hit a certain level, the cells could undergo an oncologic change [26]. Thus, RNF180 PM is associated with the transformation of gastric cancer.

We therefore propose that AMR could be a biomarker for gastric cancer and its precursor, AG. At
AMR $>0.153$, we found the highest possibility that gastric tissue was no longer healthy, but was either gastric cancer or precancerous AG, with AMR $>0.316$ to be the best cutoff value between GC and AG. Thus, among patients who were already suffering from AG, an RNF180 PM average rate $>0.316$ could be considered an indicator of high likelihood of gastric cancer, and of a need for intervention or treatment.

We also analyzed the distribution of methylated or hypermethylated $\mathrm{CpG}$ sites count (MSC or HSC) in the $\mathrm{CON}, \mathrm{AG}$ and $\mathrm{GC}$ groups. The results showed that HSC increased gradually with disease severity $(\mathrm{GC}>\mathrm{AG}>\mathrm{CON})$, although it did not significantly differ between the $\mathrm{GC}$ and $\mathrm{AG}$ groups. We also found that MSC $>4$, among a total $31 \mathrm{CpG}$ sites, was $78.6 \%$ sensitive and $77.4 \%$ specific ( $\mathrm{AUC}=0.772$ ) in identifying $\mathrm{GC}+\mathrm{AG}$; and MSC $>15$ was $81.9 \%$ specific in predicting GC. When HSC $>1$ among the 31 targeted $\mathrm{CpG}$ sites, it was $74.5 \%$ sensitive and $83.3 \%$ specific in identifying $\mathrm{GC}$ and $\mathrm{AG}$ $(\mathrm{AUC}=0.813)$; HSC $>6$ was $92.1 \%$ specific in predicting GC. The results were consistent previous results with AMR. Beside all these results, Therefore, MSC and HSC could also be indicators of GC or GC $+\mathrm{AG}$, and generally rise with the severity of disease. Considering MSC and HSC results were shown almost the same, we advice that these results could be used according to the actual needs. For example, if we want to use lower sensitive method such as MSP or COBRA to identification GC or $\mathrm{AG}$ we could use hypermethylated $\mathrm{CpG}$ site as a biomarker for primer designing. Besides methylated CpG site can be used to achieve higher sensitivity, so that may achieve early detection of precancerous or gastric cancer.

To find $\mathrm{CpG}$ sites with hypermethylation that is most indicative of $\mathrm{AG}$ or $\mathrm{GC}$, we further selected hypermethylation occurrence frequency in gastric cancer or atrophic gastritis over than $25 \%$ among all 513 individuals are been analyzed. We found that 
Table 4: Association between H.pylori infection and the risks of Hypermethylation frequency in different gastric diseases

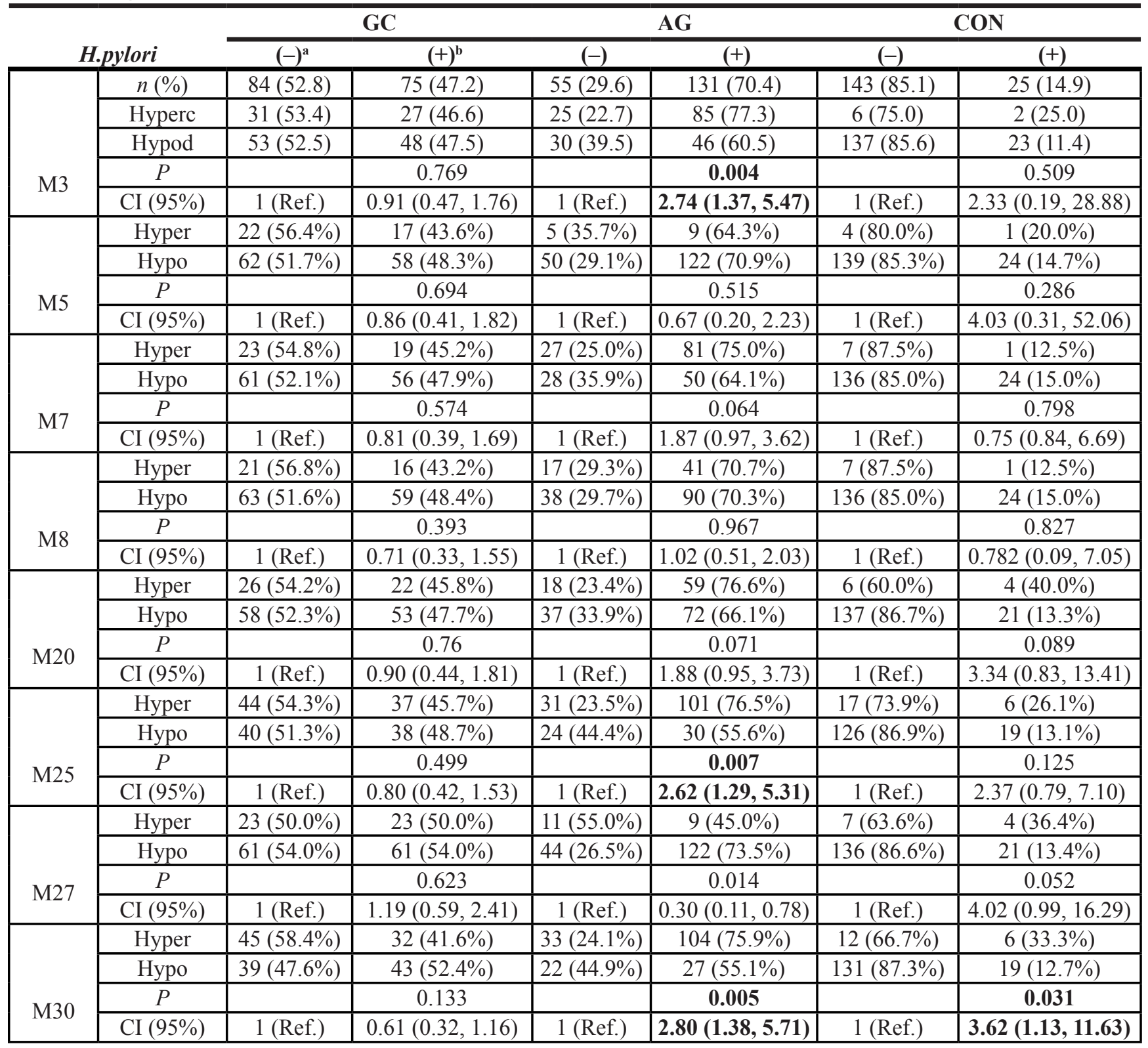

a: H.pylori infection positive. b: H.pylori infection negative. c: Hypermethylated cases d: Hypomethylated or no-methylation cases. $P$ value was adjusted by sex age smoking and drinking. $P$ value OR and corresponding $95 \%$ interval confidence were calculated to measure the association between H.pylori infection and the risks of hypermethylated CpG site. GC: gastric cancer; AG: atrophic gastritis; CON: control; OR: odd ratio; CI: interval confidence.

in $5 \mathrm{CpG}$ sites-M3(-165), M7 (-133), M20(-57), $\mathrm{M} 25(-34)$ and $\mathrm{M} 30(+5)$ - hypermethylation frequency was highest in the AG group, second-highest in the $\mathrm{GC}$ group, and lowest in the control group, and their differences were statistically significant. Besides M5(-148) and M27(-26) hypermethylation frequency was higher in GC group than Non-GC group (AG + CON). Hypermethylation of M5(-148), M27(-26) $\mathrm{CpG}$ sites were associated with 5.85 OR $(95 \% \mathrm{CI}$ : 3.20-10.69) and 4.27 OR (95\% CI: 2.54-7.19) in GC risk, respectively. Reportedly, $3 \mathrm{CpG}$ sites in the
DACT1 gene promoter are related to GC prognosis [27]. Another study showed that hypermethylation of certain CpG sites were related to AG severity [28]. Our study found $5 \mathrm{CpG}$ sites in $R N F 180$ promoter region that were related to $\mathrm{AG}$ progression, and $2 \mathrm{CpG}$ sites related to $\mathrm{GC}$ risk. These specific $\mathrm{CpG}$ sites could be used for early warning of precancerous disease or gastric cancer.

In the previous study, RNF180 transcript was identified to be specially silenced or down-regulated in gastric cancer cells and primary gastric cancer tissues, and the promoter methylation was found to directly mediate 
RNF180 transcription silencing in vitro [12]. In this study, we detected the quantitative methylation of RNF180 promoter and RNF180 mRNA expression simultaneously in gastric cancer and paired non-tumor tissues. The mRNA expressive level of RNF180 was demonstrated to be significantly lower in tumor tissues than that in non-tumor tissues, and with the same tend as which in hypermethylation tissues than that in hypomethylation tissues. The results of RNF180 gene expression were consistent with Deng's report [13], and which further suggesting that the aberrant RNF180 mRNA transcription might be resulted from the DNA promoter methylation in vivo.

H.pylori as a class I carcinogen involved in gastric carcinogenesis, and was considered to affect some of tumor suppressor genes through hypermethylation [29]. To determine whether H.pylori infection affected methylation of RNF180 promoter region, we further stratified the $\mathrm{CON}, \mathrm{AG}$ and $\mathrm{GC}$ groups by H.pylori infection to calculate differences of AMR, MSC and HSC between the H.pylori ${ }^{-}$and H.pylori ${ }^{+}$subgroups. We found that AMR and HSC in $\mathrm{CON} / H$.pylori ${ }^{+}$subgroup were higher than in the $\mathrm{CON} /$ H.pylori ${ }^{-}$subgroup. In the AG group, the M3(-165), M25(-34) and M30(+5) sites had increased hypermethylation risks of 2.74 folds $(95 \% \mathrm{CI}: 1.37-5.47)$, 2.62 folds (95\% CI: 1.29-5.31), 2.80 folds (95\% CI: 1.38-5.71), respectively, in the H.pylori ${ }^{+}$subgroup than in the H.pylori ${ }^{-}$subgroup; whereas hypermethylation of the M27(-26) site was higher in H.pylori $i^{-}$subgroup than in the H.pylori ${ }^{+}$subgroup. However, only 9 cases showed M27(-26) hypermethylated in the AG subgroup. H.pylori can release a variety of virulent determinants, such as CagA, VacA etc., that are involved in biological changes that eventually lead to AG or GC $[21,30]$. H.pylori infection can also lead to abnormal methylation of FOXD3, E-cadherin and other genes, but the methylation status of these genes can be restored after removal of the H.pylori infection, with increases in the products of these genes [17-19]. H.pylori can affect methylation in two ways: overall $\mathrm{CpG}$ site methylation, and methylation of certain single $\mathrm{CpG}$ sites [31-33]. Our results, together with those of previous reports, indicate that H.pylori infection can increase overall methylation in normal or slightly superficial gastritis tissues, but after AG develops, H.pylori can increase hypermethylation at $\mathrm{M} 3(-165), \mathrm{M} 25(-34)$ and $\mathrm{M} 30(+5)$ in the RNF180 promoter region. Thus, H.pylori infection may vary its modification of RNF180 gene PM under the different disease conditions.

This study had some limitations. First, all subjects were from northern China, and we did not include other geographical or ethnic populations, which may have affected our results. However, considering that $42 \%$ of gastric cancers occur in China [34], this study has important reference value. Second, our cohort was rather small, especially the H.pylori ${ }^{+}$subgroup with a hypermethylated RNF180 promoter. Therefore, our results should be verified and expanded with a larger and more varied study group.
In summary, this study focused on relationships between methylation of RNF180 promoter and gastric cancer or atrophic gastritis and the effect of H.pylori infection on RNF180 PM. We found, for the first time, that AMR, MSC, and HSC at particular CpG sites within $R N F 180$ promoter region could potentially be an early indicator of gastric cancer and atrophic gastritis. The aberrant expression of RNF180 mRNA levels in tumor might be resulted from the DNA promoter methylation. We also found that H.pylori infection may vary how it modifies methylation in the RNF180 gene promoter under different disease conditions. That is, in normal or superficial gastritis H.pylori tend to affect overall methylation status rather than hypermethylating certain $\mathrm{CpG}$ sites. But in atrophic gastritis, H.pylori was more likely to affect certain $\mathrm{CpG}$ sites rather than the overall methylation status. This study provides an experimental basis to evaluate $R N F 180$ promoter methylation as a biomarker for early warning and diagnosis of gastric cancer or atrophic gastritis.

\section{MATERIALS AND METHODS}

\section{Sample collection}

This study was approved by the Human Ethics Review Committee of the First Affiliated Hospital of China Medical University (Shenyang, China). All subjects gave informed consents in accordance with the declaration of Helsinki and its latest revision. We enrolled a total of 513 subjects who had undergone endoscopic examination at the Zhuanghe Gastric Diseases Screening Program and the Surgical Oncology Department in the First Affiliated Hospital of China Medical University between July, 2008 and June, 2010. Out of 513 tissue samples, 168 were normal gastric mucosa or slightly superficial gastritis tissues for the control group (CON), 186 were AG tissues, and 159 were GC tissues. In addition, we collected 55 pairs of fresh tumor tissues and non-tumor tissues from GC patients who underwent curative gastrectomy between 10, 2012 and 10, 2015 at the First Affiliated Hospital of China Medical University for RNF180 gene expression analysis. All the gastric mucosa tissues were histologically verified and their corresponding histological information was collected. Atrophic gastritis and superficial gastritis were classified by the Sydney classification system [20]. Although AG can be histologically classified as mild, moderate or severe, only moderate or severe AG were included in this study. We diagnosed CON tissues as normal stomach, or as mild superficial gastritis if mesenchymal lymphocyte counts were $<30 \%$ after excluding other systemic and gastric diseases. None of the patients had received preoperative radiotherapy or chemotherapy. We collected subjects' age, sex and other relevant clinical information by questionnaire and computerized medical records. Their GC histological diagnoses were based on the World Health Organization's criteria [16]. Participants' clinicopathological characteristics are listed in Table 5. 
Table 5: Clinicopathological characteristics

\begin{tabular}{|c|c|c|c|c|c|}
\hline & & GC (\%) & AG (\%) & CON (\%) & $P$ value \\
\hline \multirow[t]{2}{*}{ Sex } & Male & $105(66.0)$ & $112(60.2)$ & $99(58.9)$ & \\
\hline & Female & $54(34.0)$ & $74(39.8)$ & $69(41.1)$ & 0.363 \\
\hline Age & Ave. \pm Std. & $58.9 \pm 11.7$ & $53.4 \pm 8.3$ & $54.0 \pm 7.9$ & $<0.001$ \\
\hline \multirow[t]{2}{*}{ Smoking } & $(-)$ & $87(54.7)$ & $133(71.4)$ & $119(70.8)$ & \\
\hline & $(+)$ & $72(45.3)$ & $53(28.5)$ & $49(29.2)$ & 0.001 \\
\hline \multirow[t]{2}{*}{ Drinking } & $(-)$ & $104(65.4)$ & $144(77.4)$ & $129(76.8)$ & \\
\hline & $(+)$ & $55(34.6)$ & $42(22.6)$ & $39(23.2)$ & 0.022 \\
\hline \multirow[t]{2}{*}{ H.pylori } & $(-)$ & $84(52.8)$ & $55(29.6)$ & $143(85.1)$ & \\
\hline & $(+)$ & $75(47.2)$ & $131(70.4)$ & $25(14.9)$ & $<0.001$ \\
\hline Total & 513 & 159 & 186 & 168 & \\
\hline
\end{tabular}

GC: gastric cancer; AG: atrophic gastritis; $\mathrm{CON}$ :control.

\section{DNA extraction and sodium bisulfite modification}

We extracted genomic DNA from $20-\mu \mathrm{m}$ sections of paraffin-embedded gastric epithelial tissues using paraffin tissue extraction kit (TianGen Biochemistry, Beijing). Sodium bisulfite modification of DNA was preceded by use of the Zymo DNA Methylation-Gold kit (Zymo Research, US). All procedures followed the manufacturers' instructions.

\section{Enzyme-linked immunosorbent assay (ELISA) for H.pylori infection}

Serum immunoglobulin (Ig) $\mathrm{G}$ antibodies to H. pylori were detected by $H$. pylori-IgG enzyme-linked immunosorbent assay (ELISA; Biohit, Helsinki, Finland). Antibody titers were quantified by optical density readings according to manufacturer's protocol; titers > 34 EIU (threshold value) were considered positive for H.pylori infection.

\section{Bisulfite genomic sequencing}

All samples were quantitatively analyzed using bisulfite genomic sequencing (BGS) for the RNF180 promoter area. We amplified the $R N F 180$ promoter area from -224 to +94 , for a 319-bp fragment, with these primers: F: 5'-GTGGTTTTGGTAAGGGGATGATCC-3'; R: 5'-AACAACCAAACTCTAAAAACTC-3' [13]. All methylation PCRs were used HotStart Taq 2.0 Version (Takara, Japan), and these conditions: initial denaturation at $94^{\circ} \mathrm{C}$ for $3 \mathrm{~min} ; 45$ cycles $\left(94^{\circ} \mathrm{C}\right.$ for $20 \mathrm{sec}, 58.5^{\circ} \mathrm{C}$ for 30 sec, $72^{\circ} \mathrm{C}$ for $45 \mathrm{sec}$ ); followed by a final extension at $72^{\circ} \mathrm{C}$ for 10 minutes, with a final termination at $4^{\circ} \mathrm{C}$. All PCR products were electrophoresed with $2 \%$ agarose gel, and staining with Genefinder (Xiamen, Zeeshan Biotechnology); remaining PCR products that shown bright band were used for backward sequence analysis after product purification.
According to the theory of bisulfite modification, unmethylated cytosine changes into uracil, and uracil is replaced by thymine during PCR; whereas methylated cytosine remains the same. The methylation rate of forward sequencing was: Meth $\%=[\mathrm{C} /(\mathrm{C}+\mathrm{T})]^{*} 100 \%$. Therefore, complementary base pairing should show a methylation rate of reverse sequencing as: Meth\% $=[\mathrm{G} /(\mathrm{G}+\mathrm{A})]^{*} 100 \%$. The average methylation rate (AMR; the average methylation rate for all $\mathrm{CpG}$ sites in each sample) methylated (methylation rate $>0.25) \mathrm{CpG}$ sites count (MSC) and hypermethylated (methylation rate $>0.50$ ) CpG sites count (HSC) was used to quantify methylation status.

In order to further confirm credibility test result, we randomly selected 10 cases of cloning sequencing DNA samples as quality control. The purified PCR products were cloned into the pUC18-T vector (Biodee, Beijing, China), and ten clones for each sample were randomly selected and sequenced (Beijing, Genomics Biotechnology). No significant differences were found between these two methods ( $P=0.648$, Supplementary Table S2).

\section{Quantitative real-time PCR analysis of RNF180 mRNA expression}

55 pairs of gastric cancer tissues and adjacent noncancer tissues were detected the RNF180 expression by using the quantitative real-time PCR for demonstration the difference of RNF180 mRNA expression between two groups of tissues. RNA was extracted using TRIzol reagent (Life Technologies, Carlsbad, CA) according to the manufacturer's instructions. Total RNA was converted into complementary DNA using Quantscript RT kit (Tiangen Biotech, Beijing, China). The mRNA levels specific for RNF180 genes and an internalcontrol gene glyceraldehyde 3-phosphate dehydrogenase $(G A P D H)$ were examined using SYBR Premix Ex Taq II (TaKaRa Biotech, Dalian, China) according to 
the manufacturer's protocol. Primers designed and utilized for RNF180 was as follows: Forward sequence: 5'-GTGCAGTGTGTCTGGACGTT-3', and Reverse sequence: 5'-AATGGGCATGGAGTGCTTGA-3'. Primers designed and utilized for $G A P D H$ was as follows: Forward sequence: 5'-TGCACCACCAACTGCTTAG-3', and Reverse sequence: 5'-GGATGCAGGGATGATGTTCC-3'. Melting curve analysis was performed to exclude the presence of non-specific products and primer-dimers. Each reaction was performed in duplicates, and no-template controls were included in each experiment.

The relative quantification of gene mRNA expression was calculated using the $2^{-\Delta \Delta C t}$ method [21]. The expression levels of $R N F 180$ were normalized to those of GAPDH in each sample using the equation: $\triangle \mathrm{Ct}$ (delta $\mathrm{Ct})=\mathrm{Ct}_{\text {target }}-\mathrm{Ct}_{\mathrm{GAPDH}}$. Relative expression levels were derived from $\Delta \mathrm{Ct}$ values as $2^{-\Delta C t}$. The relative expression of tissues with tumor or hypermethylation were set to a unity, and the relative expression of tissues with non-tumor or hypomethylation were expressed relative to those of tissues with tumor or hypermethylation, thus deriving normalized $2^{-\Delta \Delta C t}$ values.

\section{Statistical analysis}

All statistical analysis was performed using SPSS 20.0 software (SPSS, Chicago, IL, USA). The Paired samples $t$-test was used to compare differences of the methods between Direct sequencing and Cloning sequencing. The Mann-Whitney $U$ test was used to compare differences in AMR, MSC, HSC and relative mRNA levels among different groups. Receiver operating characteristic (ROC) curves and areas under curves (AUC) were used to analyze $\mathrm{GC}, \mathrm{AG}$ and $\mathrm{CON}$ groups with regard to AMR, MSC and HSC. Multivariate logistic regression with adjustments for age, sex, smoking, drinking and H.pylori infection was used to assess associations between hypermethylation frequency of individual $\mathrm{CpG}$ sites and disease risk, and hypermethylation risk for H.pylori infection.

\section{Abbreviations}

GC: Gastric cancer; AG: Atrophic gastritis; $\mathrm{CON}$ : Control; AMR: Methylation rates; MSC: Methylated CpG site counts; HSC: Hypermethylated CpG site counts; PM: Promoter methylation; H.pylori: Helicobacter pylori. ELISA: Enzyme-linked immunosorbent assay; ROC: Receiver operating characteristic; AUC: Curves and areas under curves.

\section{GRANT SUPPORT}

This work is supported by grants from the National Key Basic Research Program of China (973 Program ref no. 2010CB529304).

\section{CONFLICTS OF INTEREST}

All authors disclose any conflicts of interest that might bias their work.

\section{REFERENCES}

1. Sugiura H, Ishiguro H, Kuwabara Y, Kimura M, Mitsui A, Mori Y, Ogawa R, Katada T, Harata K, Fujii Y. Decreased expression of RUNX3 is correlated with tumor progression and poor prognosis in patients with esophageal squamous cell carcinoma. Oncol Rep. 2008; 19:713-9. doi: 10.3892/ or.19.3.713.

2. Veeck J, Wild PJ, Fuchs T, Schuffler PJ, Hartmann A, Knuchel R, Dahl E. Prognostic relevance of Wnt-inhibitory factor-1 (WIF1) and Dickkopf-3 (DKK3) promoter methylation in human breast cancer. BMC Cancer. 2009; 9:217. doi: 10.1186/1471-2407-9-217.

3. Serizawa RR, Ralfkiaer U, Steven K, Lam GW, Schmiedel S, Schuz J, Hansen AB, Horn T, Guldberg P. Integrated genetic and epigenetic analysis of bladder cancer reveals an additive diagnostic value of FGFR3 mutations and hypermethylation events. Int J Cancer. 2011; 129: 78-87. doi: 10.1002/ijc.25651.

4. Cheng YY, Yu J, Wong YP, Man EP, To KF, Jin VX, Li J, Tao Q, Sung JJ, Chan FK, Leung WK. Frequent epigenetic inactivation of secreted frizzled-related protein 2 (SFRP2) by promoter methylation in human gastric cancer. Br J Cancer. 2007; 97:895-901. doi: 10.1038/ sj.bjc. 6603968.

5. Zhang X, Zhang X, Sun B, Lu H, Wang D, Yuan X, Huang Z. Detection of aberrant promoter methylation of RNF180, DAPK1 and SFRP2 in plasma DNA of patients with gastric cancer. Oncol Lett. 2014; 8:1745-50. doi: 10.3892/ol.2014.2410.

6. Mehta A, Dobersch S, Romero-Olmedo AJ, Barreto G. Epigenetics in lung cancer diagnosis and therapy. Cancer Metastasis Rev. 2015; 34:229-41. doi: 10.1007/ s10555-015-9563-3.

7. Langevin SM, Kratzke RA, Kelsey KT. Epigenetics of lung cancer. Transl Res. 2015; 165:74-90. doi: 10.1016/j. trs1.2014.03.001.

8. Fanelli M, Fantozzi A, De Luca P, Caprodossi S, Matsuzawa S, Lazar MA, Pelicci PG, Minucci S. The coiled-coil domain is the structural determinant for mammalian homologues of Drosophila Sina-mediated degradation of promyelocytic leukemia protein and other tripartite motif proteins by the proteasome. J Biol Chem. 2004; 279:5374-9. doi:10.1074/jbc.M306407200.

9. Balastik M, Ferraguti F, Pires-da Silva A, Lee TH, AlvarezBolado G, Lu KP, Gruss P. Deficiency in ubiquitin ligase TRIM2 causes accumulation of neurofilament light chain and neurodegeneration. Proc Natl Acad Sci U S A. 2008; 105:12016-21. doi: 10.1073/pnas.0802261105. 
10. Neumuller RA, Betschinger J, Fischer A, Bushati N, Poernbacher I, Mechtler K, Cohen SM, Knoblich JA. Mei-P26 regulates microRNAs and cell growth in the Drosophila ovarian stem cell lineage. Nature. 2008; 454:241-5. doi: 10.1038/nature07014.

11. Ogawa M, Mizugishi K, Ishiguro A, Koyabu Y, Imai Y, Takahashi R, Mikoshiba K, Aruga J. Rines/RNF180, a novel RING finger gene-encoded product, is a membranebound ubiquitin ligase. Genes Cells. 2008; 13:397-409. doi: 10.1111/j.1365-2443.

12. Cheung KF, Lam $\mathrm{CN}, \mathrm{Wu} \mathrm{K}, \mathrm{Ng} \mathrm{EK}$, Chong WW, Cheng AS, To KF, Fan D, Sung JJ, Yu J. Characterization of the gene structure, functional significance, and clinical application of RNF180, a novel gene in gastric cancer. Cancer. 2012; 118:947-59. doi: 10.1002/cncr.26189.

13. Deng J, Liang H, Ying G, Zhang R, Wang B, Yu J, Fan D, Hao X. Methylation of CpG sites in RNF180 DNA promoter prediction poor survival of gastric cancer. Oncotarget. 2014; 5:3173-83. doi: 10.18632/oncotarget. 1888

14. Xie X, Deng J, Hou Y, Cui J, Wu W, Ying G, Dong Q, Hao X, Liang H. Evaluating the clinical feasibility: The direct bisulfite genomic sequencing for examination of methylated status of E3 ubiquitin ligase RNF180 DNA promoter to predict the survival of gastric cancer. Cancer Biomark. 2015; 15:259-65. doi: 10.3233/CBM-150466.

15. Bhardwaj V, Noto JM, Wei J1, Andl C, El-Rifai W, Peek RM, Zaika AI. Helicobacter pylori bacteria alter the p53 stress response via ERK-HDM2 pathway. Oncotarget. 2015; 6:1531-43. doi: 10.18632/oncotarget.2828.

16. Zaika A, Wei J, Noto J, Peek R, Jr. Regulation of the p53 tumor suppressor by gastric pathogen Helicobacter pylori pathogen. Oncotarget. 2012; 3:1057-8. doi: 10.18632/ oncotarget.698.

17. Cheng AS, Li MS, Kang W, Cheng VY, Chou JL, Lau SS, Go MY, Lee CC, Ling TK, Ng EK, Yu J, Huang TH, To KF, et al. Helicobacter pylori causes epigenetic dysregulation of FOXD3 to promote gastric carcinogenesis. Gastroenterology. 2013; 144:122-33. doi: 10.1053/ j.gastro.2012.10.002.

18. Leung WK, Man EP, Yu J, Go MY, To KF, Yamaoka Y, Cheng VY, Ng EK, Sung JJ. Effects of Helicobacter pylori eradication on methylation status of E-cadherin gene in noncancerous stomach. Clin Cancer Res. 2006; 12:3216-21. doi: 10.1158/1078-0432.CCR-05-2442

19. Chan AO, Peng JZ, Lam SK, Lai KC, Yuen MF, Cheung HK, Kwong YL, Rashid A, Chan CK, Wong BC. Eradication of Helicobacter pylori infection reverses E-cadherin promoter hypermethylation. Gut. 2006; 55:463-8. doi: 10.1136/ gut.2005.077776.

20. Dixon MF, Genta RM, Yardley JH, Correa P. Classification and grading of gastritis. The updated Sydney System. International Workshop on the Histopathology of Gastritis, Houston 1994. Am J Surg Pathol. 1996; 20:1161-81.

21. He C, Tu H, Sun L, Xu Q, Li P, Gong Y, Dong N, Yuan Y. Helicobacter pylori-related host gene polymorphisms associated with susceptibility of gastric carcinogenesis: a two-stage case-control study in Chinese. Carcinogenesis. 2013; 34:1450-7. doi: 10.1093/carcin/bgt079.

22. Baylin SB, Ohm JE. Epigenetic gene silencing in cancer a mechanism for early oncogenic pathway addiction? Nat Rev Cancer. 2006; 6:107-16. doi:10.1038/nrc1799

23. Jones PA, Baylin SB. The fundamental role of epigenetic events in cancer. Nat Rev Genet. 2002; 3:415-28. doi:10.1038/nrg816.

24. Esteller M. CpG island hypermethylation and tumor suppressor genes: a booming present, a brighter future. Oncogene. 2002; 21:5427-40. doi:10.1038/sj.onc. 1205600.

25. Dammann R, Schagdarsurengin U, Seidel C, Strunnikova M, Rastetter M, Baier K, Pfeifer GP. The tumor suppressor RASSF1A in human carcinogenesis: an update. Histol Histopathol. 2005; 20:645-663.

26. Chen HY, Zhu BH, Zhang CH, Yang DJ, Peng JJ, Chen JH, Liu FK, He YL. High CpG island methylator phenotype is associated with lymph node metastasis and prognosis in gastric cancer. Cancer Sci. 2012; 103:73-9. doi: 10.1111/ j.1349-7006.2011.02129.x.

27. Deng J, Liang H, Zhang R, Ying G, Xie X, Yu J, Fan D, Hao X. Methylated CpG site count of dapper homolog 1 (DACT1) promoter prediction the poor survival of gastric cancer. Am J Cancer Res. 2014; 4:518-27.

28. Tahara T, Arisawa T, Shibata T, Nakamura M, Yoshioka D, Okubo M, Maruyama N, Kamano T, Kamiya Y, Fujita H, Nakagawa Y, Nagasaka M, Iwata $M$, et al. Increased number of methylated $\mathrm{CpG}$ islands correlates with Helicobacter pylori infection, histological and serological severity of chronic gastritis. European journal of gastroenterology \& hepatology. 2009; 21:613-9. doi: 10.1097/MEG.0b013e32830e28b2.

29. Zhang X, Zhang X, Sun B, Lu H, Wang D, Yuan X, Huang Z. Detection of aberrant promoter methylation of RNF180, DAPK1 and SFRP2 in plasma DNA of patients with gastric cancer. Oncol Lett. 2014; 8:1745-50. doi: 10.3892/ ol.2014.2410.

30. Mégraud F, Bessède E, Varon C. Helicobacter pylori infection and gastric carcinoma. Clin Microbiol Infect. 2015; 21:984-90. doi: 10.1016/j.

31. Ling ZQ, Ge MH, Lu XX, Han J, Wu YC, Liu X, Zhu X, Hong LL. Ndrg2 promoter hypermethylation triggered by helicobacter pylori infection correlates with poor patients survival in human gastric carcinoma. Oncotarget. 2015; 6:8210-25. doi: 10.18632/oncotarget.3601.

32. Rodriguez-Paredes M, Esteller M. Cancer epigenetics reaches mainstream oncology. Nature medicine. 2011; 17:330-9. doi: 10.1038/nm.2305.

33. Taby R, Issa JP. Cancer epigenetics. CA Cancer J Clin. 2010; 60:376-92. doi: 10.3322/caac.20085.

34. World Health Organization. Fact Sheet No 297, Cancer. 2009. 